\title{
CONTRIBUCIÓN A UNA CRÍTICA EPISTEMOLÓGICA DE LA DISCAPACIDAD INTELECTUAL
}

\author{
MARÍA CECILIA TAMBURRINO \\ Universidad de Buenos Aires, Argentina
}

Recibido: 04/11/2008

Aceptado: 03/03/2009

\section{Introducción}

Con la intención de contribuir al esclarecimiento de las complejas relaciones entre institucionalización y discapacidad intelectual, ${ }^{1}$ en este artículo se realiza un ejercicio de indagación epistemológica del «retraso mental»y su criterio diagnóstico principal, el déficit en la inteligencia.

Para ello, tomaré como insumo los avances de una investigación etnográfica en curso donde analizo los procesos sociales, políticos, culturales, económicos, vinculares y subjetivos involucrados en las prácticas y las políticas de institucionalización de personas con discapacidad intelectual en un hospital psiquiátrico del Área Metropolitana de Buenos Aires, Argentina.

A lo largo del trabajo apreciaremos al diagnóstico como una técnica política que involucra dimensiones morales y epistémicas. Al mismo tiempo, daremos cuenta del modo en que el déficit intelectual y la valoración diagnóstica dan lugar a la institucionalización de los sujetos con discapacidad intelectual.

En un primer momento, entonces, reconstruiré los elementos que componen el cuadro diagnóstico desde la perspectiva de los profesionales del hospi-

1. Utilizo el término discapacidad intelectual para referirme de manera genérica a esta población. «Retraso mental» además de ser una categoría nativa, es el término utilizado por las clasificaciones y manuales psiquiátricos para referirse a este diagnóstico.

Feminismo/s 13, junio 2009, pp. 187-206 
tal; apreciaremos el alto contenido moral y los valores epistemológicos que se exponen en las descripciones.

En un segundo momento, indagaré en las moralidades y presupuestos epistemológicos que están en juego en la definición de la inteligencia. La inteligencia produce una cadena de sentido donde se ubica no sólo al lenguaje y al pensamiento abstracto, lógico y racional, sino a la voluntad, el juicio, la conciencia, la moral y la autonomía.

La inteligencia se revela como un concepto confuso que abarca un extenso conjunto de dimensiones y dominios. Mostraremos que esta imprecisión y flexibilidad conceptual contribuye a la colonización de toda la vida del sujeto portador del déficit: sus afectos, emociones, sus habilidades, saberes, sus acciones, gestos, movimientos, posturas, disposiciones, lenguajes, su voluntad, su juicio, su moral y su posibilidad de ser autónomo.

El análisis de la dimensión epistemológica de la valoración diagnóstica del «retraso mental» nos permite mostrar que más que una cualidad o atributo, la inteligencia es una tecnología política con particulares prácticas, discursos, procedimientos y saberes. La tecnología política de la inteligencia opera diferenciando seres humanos y produciendo desigualdad social: la inteligencia ha sido uno de los medios de justificación y legitimación del confinamiento de las mujeres a la esfera privada, así como de las poblaciones de origen africano a la esclavitud y de los indígenas al colonialismo.

Veremos que un aspecto fundamental de la inteligencia es el género. Apreciaremos el modo en que la tecnología política de la inteligencia ha servido para fundamentar y validar las desigualdades sexo-genéricas: la inteligencia es uno de los medios en que se reproducen los fundamentos epistemológicos androcéntricos de la sociedad capitalista, tales como la abstracción, el lenguaje, la razón, la madurez, la conciencia, la independencia, entre otros.

Desde una perspectiva etnográfica, y en un marco de observación participante, desde marzo de 2007 estoy llevando a cabo el trabajo de campo en un hospital psiquiátrico para varones ubicado en el Área Metropolitana de Buenos Aires, Argentina. Inicié mis tareas de campo en seis servicios diferentes pero aquí trabajo con materiales provenientes de tres servicios de internación de largo plazo - «servicio de crónicos»-. El conjunto de los profesionales ${ }^{2}$ está compuesto por psicólogos, psicoanalistas y psiquiatras. Son catorce profesionales en total (seis mujeres y ocho varones). El análisis de este trabajo se llevará a cabo con notas de campo de la observación participante, entrevistas

2. Con el fin de hacer más sencilla la lectura se utiliza el masculino como genérico. Sin embargo, debe recordarse que los profesionales incluye a varones y a mujeres; no así los pacientes que son todos varones. 
a profesionales y análisis de las historias clínicas ${ }^{3}$ que giran en torno de la vida de diecisiete pacientes con diagnóstico de «retraso mental» que viven en la institución. Llevan internados en promedio más de 20 años: los años de ingreso oscilan entre 1965 y 2000.

\section{La discapacidad intelectual}

Oligofrenia, deficiencia mental, retraso mental, debilidad mental, discapacidad mental, discapacidad cognitiva, discapacidad intelectual, etcétera, son diferentes nombres utilizados para identificar un mismo diagnóstico:

El grupo de alteraciones psíquicas determinadas por un desarrollo intelectual insuficiente, de origen congénito o en períodos precoces de la vida. Clásicamente se oponen a las Demencias, que son deterioros de una inteligencia que ya se había desarrollado normalmente a lo largo de la existencia del sujeto ${ }^{4}$.

La inteligencia siempre ha sido la noción central en el «retraso mental». De origen congénito o adquirido en los primeros años de vida, las diferentes definiciones y criterios diagnósticos de la psiquiatría y la psicología tienen por referencia común la delimitación de la esfera intelectual como la instancia -deficitaria- que marca la especificidad del diagnóstico.

Desde principios del siglo Xx, el criterio psicométrico 5 es el más utilizado no sólo para la medición de la inteligencia sino como criterio diagnóstico del «retraso mental». La inteligencia se mide cuantificando la proximidad o desviación respecto de los valores considerados normales según la edad del individuo: el coeficiente intelectual (CI). Los valores del CI son un índice relativo a la edad: en una curva de distribución normal, la media de cada edad tiene siempre el valor $\mathrm{CI}=100$ y una desviación estándar de puntuación que corresponde a 15 puntos $\mathrm{CI}^{6}$. Por ello, el $\mathrm{CI}$ es un índice de dispersión que informa de la posición relativa de un determinado sujeto respecto a su grupo de edad normativo ${ }^{7}$. Lo que define al «retraso mental» es su desviación paramétrica y su distancia respecto de la inteligencia normal: esta distancia es objetivable y su valor es el coeficiente intelectual.

3. Algunas de las citas que aparecen en el texto pertenecen a estos contextos de enunciación (observación participante, entrevistas e historias clínicas), y aparecerán entrecomilladas y en itálica. Se indica el sujeto de la enunciación del siguiente modo: PS psicólogos y PSQ psiquiatras; HC se utiliza para hacer referencia a las historias clínicas.

4. MARTÍNEZ FERRETI, J. «Breve guía diagnóstica y pronóstica de los retrasos mentales». Alcmeon, Revista Argentina de Clínica Neuropsiquiátrica, 8, 2 (1999) p. 158.

5. La psicometría es la disciplina que se encarga de las mediciones psicológicas.

6. HORMigO, A. K. Retraso mental en niños y adolescentes. Buenos Aires, Noveduc, 2006.

7. HUNT, E. «The status of the concept of intelligence». Japanese Psychological Research, 39, 1 (1997), pp. 1-11.

Feminismo/s 13, junio 2009, pp. 187-206 
Así, sabemos que «los sujetos con expectativas de normalidad obtienen un CI que oscila entre 90 y 110, considerándose Retrasados Mentales a aquellos individuos con un CI inferior a 70. Valores entre 70 y 90 constituyen "estados límite" » ${ }^{8}$. Los distintos grados del «retraso mental» dependen de los valores del CI: entre 50-55 a 70-75 «retraso mental leve», 35-40 a 50-55 «retraso mental moderado», 20-25 a 35-40 «retraso mental grave» y por último, el «retraso mental profundo» donde el CI es inferior a 20-25.

\section{La valoración diagnóstica del «retraso mental»}

En el hospital, el principal criterio diagnóstico del «retraso mental» es el déficit en la inteligencia. Los diversos profesionales acuerdan que en un diagnóstico de este tipo «lo que cuenta es la inteligencia» (PS): sea porque falta, o sea porque está disminuida, «los débiles tienen afectada la inteligencia» (PS).

Para algunos profesionales, el «retraso mental» tiene un determinante biológico a nivel cerebral. El «retraso» es «una falla en la maduración cerebral» (PSQ), se trata de un cerebro «que no terminó de desarrollarse» (PSQ) o de un desarrollo «que no existió nunca» (PS) y por ende el sujeto «está detenido en etapas evolutivas inferiores» (PS). Desde esta perspectiva, las causas de los «retrasos mentales» son -en su mayoría- congénitas, hereditarias u orgánicas. Por ello, el déficit en la inteligencia acompaña toda la vida del sujeto «desde el nacimiento» (PSQ).

Otros profesionales, además de las causas congénitas y hereditarias y la explicación biológica, sostienen en sus argumentaciones que incluyen factores afectivos, culturales e históricos. Así, etiologías del «retraso mental»son también la falta de afecto y estimulación adecuada durante la primer época del desarrollo; el alcoholismo de los padres; golpes, traumatismos y maltrato durante la infancia, intoxicaciones, desnutrición materna y/o infantil, hasta una «sociedad que abandona» (PS), y contextos sociales adversos y empobrecidos.

Ya sea la causa una lesión orgánica, una falla genética, falta de estimulación durante el desarrollo, carencias afectivas en los primeros meses de vida, contaminación ambiental o maltrato infantil, tanto el texto de las historias clínicas como el relato de los entrevistados coinciden en que el cuadro es crónico e irreversible. Ninguno de los tratamientos, por ende, apunta a la cura porque -tratándose de un déficit en la inteligencia o una detención en el desarrollo- «no se puede curar lo que no está» (PSQ). El pronóstico «dadas las características de irreversibilidad del cuadro» (HC) es siempre «desfavorable»

8. MARTíNEZ FERRETI, José. Op. cit., p. 160.

Feminismo/s 13, junio 2009, pp. 187-206 
(HC), cuando no «malo» (HC), la «incapacidad es 100\% permanente» (HC) y la «aptitud laboral incapacitante actualmente y en el futuro» (HC).

En las historias clínicas relevadas, además de los «paciente estable», «igual medicación», y «sin modificaciones significativas para consignar» compartidas con los restantes pacientes de los servicios de «crónicos», aquellos con diagnóstico de «retraso mental» presentan otras regularidades y reiteraciones. En una misma historia clínica, y en las diferentes historias clínicas de los distintos pacientes con el mismo diagnóstico se encuentran las mismas afirmaciones:

Pensamiento de curso lento y retardado (o enlentecido) - Puerilidad, conducta pueril, pensamiento pueril - Pensamiento concreto - Inteligencia concreta - Nivel intelectual bajo - Entendimiento disminuido - Capital ideativo pobre - Asociaciones de ideas enlentecidas - Atención intelectual pobre - Labilidad - Juicio insuficiente - Bradilalia ${ }^{9}$ - Ecolalia ${ }^{10}$ - Ecomimia ${ }^{11}$ - Ecopraxia $^{12}$ - Estereotipias ${ }^{13}$ - Hipolucidez o conciencia no lúcida - Hipoprosexia $^{14}$ - Hipobulia ${ }^{15}$.

La descripción de los sujetos está sobresaturada de los prefijos «eco», «hipo», «dis», «in», «a»: la repetición, la disminución y el déficit. Estos pacientes repiten automáticamente gestos, movimientos, palabras, mímicas (ecolalia, ecomimia...); no tienen palabra propia sino «mimetización con discursos ajenos» (PS), tienen un discurso «estereotipado» (PS) y «repetitivo» (PS), movimientos semiautomáticos, patrones repetitivos del movimiento, etcétera. Y presentan déficits o disminución en la atención, en la voluntad, en el entendimiento, en el capital ideativo, en el juicio...

Desde la perspectiva profesional, entonces, el déficit intelectual provoca que el pensamiento sea de carácter concreto y pueril, con una velocidad de procesamiento de la información lenta o retrasada, con una disminuida capacidad de entendimiento y comprensión, y un juicio «insuficiente»o «debilitado» (HC). Es posible desprender ciertas características y cualidades de la inteligencia a partir de los efectos que su ausencia produce. Pues desde la perspectiva profesional, la inteligencia se caracteriza precisamente por lo contrario de las descripciones diagnósticas: la inteligencia se caracteriza por

9. Lentitud anormal del lenguaje.

10. Repetición involuntaria de una palabra o frase que acaba de pronunciar otra persona.

11. Repetición automática de los actos, gestos, etc.

12. Repetición mimética, de los gestos realizados por un interlocutor, movimientos semiautomáticos e incontrolados.

13. Conductas motoras repetitivas de alta frecuencia, que no parecen tener un propósito aparente, patrones repetitivos del movimiento.

14. Déficit de atención.

15. Deficiencia en la voluntad.

Feminismo/s 13, junio 2009, pp. 187-206 
lo abstracto y no lo concreto, por lo adulto y no lo pueril, por lo rápido y no lo lento, por la fortaleza y no la debilidad, por la creación y no la repetición, por la conciencia y no el automatismo, por la autonomía y no la dependencia.

El intelecto humano, entonces, tiene la capacidad de «generalizar, deducir, inducir» (PSQ), de «imaginar algo que no está» (PS), conceptualizar la «idea de que hay un futuro, un perro no tiene esa idea» (PSQ) y de "solucionar problemas nuevos que se le presentan al individuo» (PSQ). La inteligencia posibilita el entendimiento, la comprensión, la abstracción, la creación, la invención, la racionalidad, la «capacidad judicativa» del sujeto («establecer juicios de valor y juicios de realidad»-PSQ-). La inteligencia se materializa en «cosas prácticas» como «leer, escribir y realizar operaciones matemáticas» (PS).

Cuando la inteligencia falla, el lenguaje se ve afectado de varias maneras. Por un lado, en la posibilidad misma de adquirirlo: algunos no pueden «aprender a hablar por insuficiencia del aparato, no del aparato fonador, sino del aparato cerebral» (PSQ). Por otro, el déficit intelectual ocasiona una disminución en el rendimiento lingüístico: «Muchas veces el débil mental, y otros grados de imbecilidad y de idiocia, tienen taras físicas, dificultad para hablar, tartamudez, dislalia» (PSQ). Y por último, limitaciones en la capacidad de abstracción y razonamiento limitan el campo de representaciones lingüísticas: «si las ideas son limitadas, sobre todo en el terreno de lo abstracto, el lenguaje también es limitado, para expresar ideas abstractas, para generalizar, para deducir, para inducir» (PSQ).

Si bien según los profesionales el déficit en la inteligencia se manifiesta sobre todo «en las cuestiones abstractas y los juicios de valor» (PSQ), "al estar afectada la inteligencia, está afectada la afectividad y la actividad» (PSQ). Es decir, esta falta irradia su impronta deficitaria hacia la vida afectiva y práctica de los sujetos, es «o sea, una cosa global» (PS): significa ser «incapaz de llevar a cabo las actividades mínimas relacionadas con su subsistencia como la alimentación, la higiene y los cuidados»; a la vez que «los afectos son más primitivos, están proclives a reacciones a veces explosivas, desmedidas» (PSQ), por ello los pacientes con «retraso mental» no pueden establecer «vínculos duraderos, importantes, verdaderos» (PSQ).

Siguiendo la argumentación de los profesionales, la falta de inteligencia ocasiona "fallas en la voluntad, no hay nunca una voluntad sostenida hacia la consecución de un fin» (PSQ). Al disminuir la inteligencia diminuyen no sólo los niveles volitivos del sujeto, sino su posibilidad de ejercer un «control racional» (PS) de los instintos e impulsos. Este «bajo control racional» provoca un debilitamiento del control y del dominio de sí y por ello en la vida afectiva y práctica de los sujetos predominan la «impulsividad» (PSQ), las «cosas 
muy primarias» (PS), «instintivas» (PS), la «agresividad incontrolada» (PSQ), la «afectividad primitiva» (PS).

Como puede desprenderse de las narraciones de los profesionales, moral e inteligencia se vuelven inescindibles: al disminuir la inteligencia se reduce de modo directamente proporcional la capacidad moral del sujeto. Las aptitudes morales deficientes o inexistentes son explicadas por los efectos de la falta de inteligencia: pensamiento concreto, fallas en la voluntad, bajo control racional, debilitamiento del autodominio. La imposibilidad de desprenderse de los datos de la experiencia concreta, sensorial, impide alcanzar grados de universalización y abstracción que serían imprescindibles en el mundo de la moral. «No comprenden o comprenden mal los conceptos éticos por lo abstractos de éstos, como el deber, el bien» (PSQ).

El conjunto de caracteres que da forma al «retraso mental» explica las dificultades en la integración de estos sujetos al mundo social «normal» (PS). A partir de una falta -el déficit intelectual- no sólo se ven afectadas las «actividades mínimas relacionadas con su subsistencia», sino toda posibilidad de adaptación e inclusión a la vida en la comunidad.

No se pueden desarrollar como una persona aparentemente normal... No se pueden casar, no pueden tener hijos, etcétera» (PS)

Tener hijos me parece como demasiada responsabilidad, como que es un exceso para un paciente con estas características, por ahí una pareja de sus mismas características sí, pero tener hijos, me parece ya como mucho, ya es como mucho... Digo, si hay cosas básicas que no pueden manejar, me parece que para un hijo, no, no lo veo, cosas básicas como marcar un teléfono, manejar el dinero, lo que no tuvieron no lo pueden transmitir... digo, si vos no tuviste escolaridad no podes transmitirla» (PS)

Al no contar con los recursos intelectuales que permiten el autodominio y el control racional de los impulsos e instintos, disminuida o ausente su voluntad y su capacidad de juicio, impedido el ingreso al mundo de la moral, el sujeto con «retraso mental» queda destinado a una situación de heteronomía y dependencia.

Para mi el tema de la inteligencia debería estar relacionado con el tema de la autonomía y la dependencia y la independencia. Porque una persona con problemas intelectuales importantes, para poder autoabastecerse y tener un grado de autonomía máxima necesita mucho una dependencia de otros, o de un contexto, o de un cuidado, o de otros que pudieran colaborar en esa independencia, autonomía. En ese sentido uno podría decir que debilidad mental implica, y en muchas otras patologías también, un grado importante de dependencia (PS).

La heteronomía es modelada y definida a través de cada elemento de la descripción del cuadro. Y precisamente, la institución «viene a apuntalar, a suplir 
ese bache» (PS), «lo que no está»: el déficit. El diagnóstico mismo conlleva su pronóstico: heteronomía crónica e irreversible; es necesaria la institucionalización. «Su pronóstico es malo, atento a lo crónico e irreversible del cuadro. Es necesaria su internación» (HC).

Estos caracteres que dan forma al «retraso mental» constituyen un conjunto de sólidas certezas compartidas por los diversos profesionales. Estas sólidas certezas ocasionan que el «retraso mental» no despierte interés alguno: no representa ningún desafío, inquietud o enigma terapéutico. «Sobre el retraso mental no hay debate, no se discute, ya se sabe» (PSQ), «todo el mundo esta de acuerdo» (PSQ), «no se debate ya la etiología del cuadro, las etiologías están establecidas» (PS). Se sabe que en términos de tratamiento «no hay mucha vuelta que darle» (PS), que los tratamientos no apuntan a la cura, se sabe que es necesario que vivan «supervisados en instituciones» (PS). Se sabe cuáles son las consecuencias del «retraso mental» para el sujeto, se sabe que es un cuadro crónico e irreversible, se sabe que el pronóstico es desfavorable en todos los casos «aunque puedan funcionar medianamente bien» (PS). Se sabe lo que falta, se sabe lo que no pueden. "No se esperan cambios en su cuadro de base» (HC) o mejorías: a lo sumo, más deterioro.

Desde el nacimiento y para toda la vida, es siempre la misma monotonía de lo que falta. No hay desafíos, no hay enigmas, no hay desacuerdos ni debates; sólo sólidas certezas.

\section{La inteligencia y sus valores}

El criterio diagnóstico -y el principio de la cadena significante- del «retraso mental» es la falta de inteligencia. Sin embargo, al preguntar explícitamente a los profesionales «qué es la inteligencia», no he encontrado consenso y en ocasiones, tampoco respuesta. La noción de inteligencia no tiene un sentido unívoco entre los distintos profesionales, y tampoco en un mismo profesional.

Por un lado, un conjunto de profesionales definen la inteligencia como «la capacidad del intelecto para resolver problemas nuevos» (PSQ). En este sentido, la inteligencia se opone al instinto, definido como «el saber no aprendido de la especie» (PSQ). Asociada en sus discursos a la lógica racional y la capacidad de abstracción, la inteligencia incluye la capacidad de seriación, generalización, clasificación, inducción, deducción, etc. Otras definiciones se vinculan con la capacidad adaptativa y de funcionamiento social de los sujetos: ser inteligente es ser capaz de funcionar en el entorno social, cumpliendo con las exigencias del medio (poder llevar adelante una pareja, una familia, el cuidado de los hijos). También circulan concepciones de inteligencias múltiples (inteligencia práctica, inteligencia matemática, inteligencia musical, etc.) 
y esbozos de definiciones que vinculan la inteligencia con cuestiones culturales: la inteligencia es definida por ciertos patrones culturales al interior de un grupo dado. En otras ocasiones, la respuesta de los profesionales ante mi pregunta sobre «qué es la inteligencia» fue un prolongado silencio seguido de aclaraciones de por qué no podían contestar: «fue hace mucho que lo estudié, ahora no me acuerdo» (PS), «tendría que leer sobre el tema» (PS), entre otras.

Aunque llamativo, no es del todo sorprendente. La inteligencia ha resultado siempre un concepto complejo y confuso. A través de la revisión de la literatura sobre la temática he encontrado que hasta el momento no existe ningún acuerdo teórico, conceptual ni metodológico en las distintas disciplinas que la estudian (psicología, psiquiatría, psicopedagogía, neurología, por ejemplo). La desproporción entre la gravitación y la valoración atribuida a la inteligencia y su vaguedad e imprecisión conceptual es elocuente ${ }^{16}$. Por ejemplo, Alfred Binet -co-creador en 1905 del primer test de inteligencia de la historia- ante la pregunta sobre qué es la inteligencia contestó de modo circular: inteligencia «es lo que los tests de inteligencia miden ${ }^{17}$. Weschler, creador de una de las escalas de medición de la inteligencia con mayor vigencia en la actualidad -test de WAIS-, afirmaba que es imposible conocer la naturaleza de la inteligencia, y que sólo «conocemos la inteligencia por lo que nos permite hacer» ${ }^{18}$ :

La inteligencia general, como la electricidad, puede tomarse como una clase de energía. no sabemos cuál es la naturaleza última de esta energía, pero como en el caso de la electricidad, la conocemos por las cosas que hace, o, mejor, por las cosas que nos permite hacer -tales como hacer asociaciones apropiadas entre los eventos, derivar inferencias correctas de las proposiciones, entender el significado de las palabras, resolver problemas matemáticos o construir puentes ${ }^{19}$.

La historia del desacuerdo sobre la inteligencia comienza en simultáneo con los primeros intentos por definirla y medirla según criterios científicos, a finales del siglo XIX. A partir de entonces, numerosas han sido las definiciones de la inteligencia. Las diversas acepciones varían según el autor y la perspectiva disciplinar adoptada, y difieren en la centralidad que adquieren diferentes habilidades en detrimento de otras, en los elementos que son considerados para su análisis y en la forma de medirla. Los diversos intentos por unificar

16. Lentini, E. Discapacidad Mental. Un análisis del discurso psiquiátrico. Buenos Aires, Lugar Editorial, 2007.

17. Weschler, D. La medida de la inteligencia del adulto. Buenos Aires, Ed. Preliminar (S/F), p. 3.

18. Op. cit., p. 5.

19. Ibíd.

Feminismo/s 13, junio 2009, pp. 187-206 
criterios y consensuar definiciones, fueron infructuosos: su naturaleza, estatuto y rasgos siguen siendo motivos de debate y desacuerdo.

Pese a la dispersión conceptual pueden ser identificados una serie de rasgos o cualidades que delinean cierta unidad teórica diferencial de lo que se conoce como «versión moderna de la inteligencia» ${ }^{20}$. Por ejemplo, la relación con el lenguaje. Ya Alfred Binet creía que el poder sobre las palabras era uno de los indicadores más confiables de la presencia de inteligencia. La naturaleza y la extensión de la vinculación entre inteligencia y lenguaje han suscitado amplios debates. Para algunos, inteligencia y lenguaje son virtualmente equivalentes, otros consideran que existe una conexión estrecha pero no igualdad, otras posiciones afirman que la conexión entre inteligencia y lenguaje está dada por intermediación de la capacidad de razonamiento ${ }^{21}$.

En la literatura especializada, se considera que tanto la aptitud para el lenguaje verbal como el lenguaje matemático son rasgos distintivos de la inteligencia humana. Lenguaje y matemática denotan el valor diferencial de la capacidad de abstracción en el concepto de inteligencia. Precisamente, la capacidad de razonamiento lógico, de abstracción y la velocidad en el procesamiento de la información son tres de las habilidades más mencionadas en las definiciones de la inteligencia.

En estas definiciones, la inteligencia es inseparable de los valores asociados con el pensamiento racional y los principios de la lógica formal. Por ello «los trastornos intelectuales afectan, sobre todo, la capacidad de abstracción, síntesis y comprensión. La falta de abstracción lleva a la identificación de los objetos por datos concretos sensoriales, más que por conceptos o ideas de clase o función $»^{22}$. Y un sujeto con déficit intelectual «es incapaz de distinguir lo esencial de lo no esencial, y suele ser impulsado principalmente por los estímulos intensos, el ruido más fuerte, la luz o el color más brillante, etc. sea lo que fuere lo que percibe, se aferra a ello y su pensamiento se centra frecuentemente alrededor de estas impresionas sensoriales concretas ${ }^{23}$. La actividad mental queda circunscripta al plano de lo concreto, lo sensible y lo inmediato, pues «resulta notoriamente deficiente la elevación al grado de

20. GOODEY, C. F. «Intellectual ability and speed of performance: Galen to Galton» Science History Publications, XLII (2004).

21. Hollingworth, L. S. "Vocabulary as a symptom of intellect". American Speech, 1, 3 (1925), pp. 154-158; BOYLE, J. "Intelligence, reasoning and language proficiency". The modern language Journal, 71 (1987).

22. MARTINEZ FERRETI, J. Op. cit., p. 171.

23. MaYer-Gross et al., 1968, citado en Lentini, E. Op. cit. p. 56. 
concepto y el conferir expresión general abstracta a la multiplicidad de datos proporcionados por los sentidos» ${ }^{24}$.

Por otro lado, los comportamientos que son considerados inteligentes son altamente dependientes de criterios sociales. Se considera inteligente toda conducta que se ajusta a los principios del funcionamiento social y cultural de la sociedad. Por ello, en los estudios sobre la inteligencia, ésta se halla fuertemente asociada a la moral: cumplir con las exigencias y requerimientos de la sociedad, tener una conducta adaptada, es ser inteligente. En consecuencia, otras características añadidas y asociadas a la inteligencia son la voluntad, el criterio, el albedrío y la conciencia ${ }^{25}$.

Así por ejemplo la «inteligencia normal» es la regida por «el pensamiento abstracto en el mundo de las ideas, el sentimiento de sí mismo desarrollado, seguro y adecuado, la autodeterminación, autodominio y autorresponsabilidad y la libre capacidad de decisión ${ }^{26}$.

Y por ello los sujetos con déficits intelectuales «carecen de responsabilidad moral», pues «un cerebro deficiente trae como consecuencia un intelecto deficiente. Un intelecto deficiente trae a su vez como consecuencia una debilitación de la voluntad y el carácter. Lo que se observa más corrientemente es sin duda la disminución o ausencia de la capacidad de autodominio» ${ }^{27}$.

Las disciplinas que se encargan del estudio de la inteligencia no han llegado a un acuerdo teórico ni metodológico. Sin embargo, las distintas definiciones tienen en común que los valores, las cualidades y -muy especialmente- los efectos de la inteligencia -lo que nos permite hacer-coinciden con los contornos de lo humano, tal como ha sido definido por la tradición filosófica y metafísica occidental ${ }^{28}$. El lenguaje, la razón, el pensamiento, la moral, el juicio, la voluntad, la conciencia y la autonomía son posibles gracias a la inteligencia.

\section{Moralidades y presupuestos epistemológicos}

Abstracto y concreto; lógica e imaginación; razón y sentimiento; fortaleza y debilidad; normal y patológico; independencia y dependencia; adultez e infancia; complejo y simple; objetivo y subjetivo; universal y particular;

24. BASH, 1965; citado en LENTINI, E. Op. cit. , p. 86.

25. TARRIDA, A. C. Inteligencias. Una integración multidisciplinaria. Barcelona, Masson, 2001; GOODEY, Op. cit; HORMigO, Op. cit.

26. LUTZ, 1968, citado en LENTINI. Op. cit.

27. Cavanagh, J., citado en Lentini. Op. cit., pp. 58 y 88.

28. Agamben, G. Homo Sacer I. El poder soberano y la nuda vida. Madrid, Editorial Nacional, 2002.

Feminismo/s 13, junio 2009, pp. 187-206 
público y privado: son algunas de los estereotipos dicotómicos que han sido cuestionados por la crítica feminista por ser valores epistémicos velados del androcentrismo ${ }^{29}$.

Las valoraciones epistémicas presentes en los argumentos diagnósticos sobre el «retraso mental» exponen sin eufemismos su alto contenido moral y androcéntrico: lo concreto, lo lento, lo bajo, lo retrasado, lo infantil, lo instintivo, lo precario, lo primitivo, lo básico, lo simple se repite automáticamente en cada descripción.

El segundo término, el desvalorizado, es el que típicamente caracteriza a las personas con discapacidad intelectual en las distintas descripciones diagnósticas: movimientos anormales» (PSQ), «disminuida capacidad racional» (PS); «bajo control de los impulsos» (PS), «débil voluntad» (PS), «capacidad de comprensión demasiado elemental y superficial»(PS), «gritos o expresiones primarias» (PS), «viven un presente inmediato, concreto» ${ }^{30}$, «memoria concreta y mecánica ${ }^{31}$, «intensa credulidad ${ }^{32}$, «mayor sugestionabilidad que los hace fácilmente instrumentables $»^{33}$, «falta de la capacidad de abstracción ${ }^{34}$, «dependientes de por vida» ${ }^{35}$.

Asimismo, el contenido moral de la valoración diagnóstica recubre íntegramente la vida del sujeto con discapacidad intelectual: el lugar donde vivir, trabajar, la posibilidad de estudiar, formar una pareja, tener hijos, casarse, la capacidad de entender, decidir, tener una conducta moral, los comportamientos que puede desplegar, las actividades que puede realizar, los afectos y vínculos que puede tener... La caracterización superpone diagnóstico médico con categorización ética, donde figura nosográfica y juicio moral se confunden ${ }^{36}$.

La población con «retraso mental» encarna el reverso irreversible de los valores morales de la epistemología capitalista: el signo de lo negativo, lo no valorado, lo desechado, lo que hay que dejar atrás o sepultar para ser adultos, civilizados. De manera general, el déficit intelectual es asimilado a una condición infantil («es como un chico, no como una persona de cuarenta y pico como tiene»-PS-) y las descripciones apelan a componentes y elementos tanto más precarios, primitivos, básicos, simples cuanto más se desciende por la escala

29. MAFFIA, D. «Conocimiento y emoción», Arbor CLXXI, 716 (2005).

30. BASH, 1965; citado en LENTINI, E. Op. cit., p. 57.

31. LUTZ, 1968, citado en LENTINI, E. Op. cit., p. 46.

32. MARTíneZ FerReTI, J. Op. cit., p. 169.

33. Op. cit., p. 170.

34. Cavanagh, J., citado en Lentini. Op. cit., p. 69.

35. MARTínez FerReti, J. Op. cit., p. 173.

36. Agamben, G. Homo Sacer III. Lo que queda de Auschwitz. Madrid, Editorial Nacional, 2002. 
intelectual llegando a una indistinción entre déficit intelectual, primitivismo $\mathrm{y}$ animalidad («se asemejan a una especie inferior»-PSQ-).

Primitivismo, animalidad, infantilismo: todo eso que debe ser abandonado en la oposición naturaleza /cultura (y sus sucedáneos) caracteriza al cuadro diagnóstico. Sea cual sea la profesión o posición ideológica, los profesionales comparten un sólido núcleo de significación sobre la discapacidad intelectual. Todos comparten un mismo encadenamiento significante y un mismo pronóstico.

El campo semántico asociado al «retraso mental» está compuesto por sólidas reiteraciones: déficit, ausencia, insuficiencia, lentitud, primitivo, primario, bajo, pueril, infantil, concreto, pobre, disminución, repetición, incapacidad, imposible, retardado, involuntario, automático, no control, no voluntad, no lucidez, no atención, desfavorable, malo, crónico, irreversible, permanente, incurable.

Al reconstruir el encadenamiento significante del «retraso mental», nos topamos siempre con una misma sucesión que va de la falta de inteligencia a la heteronomía: déficit intelectual, déficit en pensamiento abstracto, lógico y racional, déficit en el lenguaje, bajo control racional, déficits en el control de los instintos, predominio de los impulsos y automatismos en el comportamiento de los individuos; afectividad primitiva, disminución o ausencia de juicio, déficit de voluntad, disminución de la capacidad moral, mayor dependencia y mayor heteronomía.

Encerrada en la valoración diagnóstica, la cadena significante liga la falta de inteligencia a la heteronomía y la dependencia, siendo el déficit una presencia constante. Eso que falta, viene a llenarlo la institución: el dominio de sí, la voluntad, el juicio, la moral...

El operador que vehiculiza y articula estas valoraciones morales es la inteligencia: «lo que cuenta es la inteligencia». La falta de inteligencia posibilita este movimiento de homologación de distintas experiencia de vida, aptitudes y cualidades a condiciones infantiles, primitivas y cuasi animales.

La inteligencia oficia de línea de corte, delimita campos epistémicos: de un lado, el apego a la realidad sensible, la materialidad, el dato concreto y sensorial; y del otro, los grados supremos de la abstracción, la lógica, la matemática, la realidad conceptual.

\section{El género de la inteligencia}

Siguiendo los argumentos profesionales, podríamos decir que un sujeto con discapacidad intelectual es un ser biológicamente imperfecto, gobernado por sus pasiones, más cerca de lo instintivo que de lo específicamente humano, 
incapaz de los rasgos de racionalidad universal y abstracta que le permitirían ser un sujeto ético ${ }^{37}$. Sin embargo, esta fórmula -que bien podría aplicarse a la población con discapacidad intelectual- está describiendo a las mujeres.

El punto de vista androcéntrico -el del varón adulto, blanco, propietario, capaz-históricamente ha negado a las mujeres racionalidad, capacidad lógica, abstracción, universalización, objetividad, atribuyéndoles condiciones a las que les restan cualquier valor epistémico ${ }^{38}$. Así, las facultades intelectuales de los varones se caracterizarían por la abstracción, la razón, el conocimiento y la objetividad; por el contrario, las mujeres se caracterizarían por la intuición, la percepción, la imitación, las sensaciones, las emociones, y la subjetividad ${ }^{39}$. Ha sido la crítica feminista la que ha puesto de manifiesto el carácter ideológico y político de las dicotomías asimétricas y ha demostrado cómo éstas históricamente han dado forma las categorías de lo femenino y lo masculino. Sin embargo, la crítica a los valores androcéntricos no se restringe a lo específico de las relaciones entre géneros; antes bien, ha demostrado cómo los valores sexo-genéricos han permeado la noción de sujeto, de ciudadanía, de derechos, e incluso la ciencia. En este movimiento crítico, se ha desarrollado un productivo campo de indagación que tiene a la epistemología como objeto. La crítica feminista ha puesto en tela de juicio muchas de las presuposiciones básicas de la epistemología tradicional: cómo se conoce, qué se conoce, quién conoce $^{40}$. Considero que uno de los principales aportes de la epistemología crítica feminista ha sido cuestionar los supuestos y valoraciones morales que se reproducen a nivel epistemológico, y señalar a la epistemología como una fuente de reproducción de la desigualdad social.

Es por ello que el análisis de la dimensión epistemológica de la inteligencia y la valoración diagnóstica del «retraso mental» revela otros modos en que los valores androcéntricos son reproducidos. La inteligencia reitera los contenidos y estereotipos morales del androcentrismo; y es precisamente el hombre blanco, adulto y capaz la encarnadura de la inteligencia normal (y su razón, abstracción, lógica, madurez, independencia, etcétera).

Al reconstruir los elementos de la valoración diagnóstica, registramos que el último eslabón de la cadena significante -iniciada con la falta de

37. MAFFIA, D. «El vinculo crítico entre genero y ciencia».S/D.

38. MAFFiA, D. «Conocimiento y emoción». Op. cit.

39. Pérez Sedeño, E. «Retóricas sexo/género», en Perez Sedeño, Eulalia y AlCalá CorTIJO, Paloma (coord.). Ciencia y Género. Madrid, Universidad Complutense de Madrid, 2001, pp. 417-434.

40. Harding, S. Ciencia y Feminismo. Madrid, Morata, 1996; Keller, E. F. Reflexiones sobre género y ciencia .Valencia, Alfons el Magnanim, 1991; Perez Sedeño, E. y AlCALÁ CORTIJO, P. (coord.). Op.cit. 
inteligencia- es la heteronomía. El argumento político que completa la valoración epistémica es que los no inteligentes dependen de los inteligentes, incluso para sobrevivir: la mujer del varón, el niño del adulto así como el «retrasado» de los cuidados ajenos.

A la luz de lo expuesto, es posible comprender por qué uno de los modos privilegiados en que se ha justificado que las mujeres no accedan a la vida pública, a la educación, a la política, al conocimiento, la ciencia, al derecho, y por consiguiente, que no se conviertan en sujetos epistémicos, ha sido radicar el argumento en una supuesta inferioridad mental: en una deficiencia mental.

La deficiencia mental de la mujer no sólo existe sino que se hace muy necesaria; no solamente es un hecho fisiológico, es también una exigencia psicológica $^{41}$.

Al relevar la literatura sobre la falta de inteligencia en las mujeres, he documentado la reiteración de los mismos elementos que en el caso de la discapacidad intelectual y el mismo proceso de homologación con la infancia, el primitivismo y la animalidad. Las mujeres son «un colectivo disminuido en aquellas facultades intelectuales y emocionales máximos productos de la evolución humana: el poder de razonamiento abstracto y de la más abstracta de las emociones, la justicia». «La mujer está entre los animales que se mueven por instinto» y el varón por el contrario «se halla liberado de él con una capacidad de raciocinio certera ${ }^{42}$.

«Incapaces de crear, inventar» ${ }^{43}$ y condenadas a la imitación y la repetición, las mujeres - del mismo modo que la población con discapacidad intelectual- no podrían despegarse de lo sensorial y material, y serían incapaces de establecer juicios que vayan más allá de lo personal y lo subjetivo. La deficiencia mental impide nuevamente el acceso al mundo de la moral y los juicios universales: las mujeres son en términos morales «evolutivamente inferiores» ${ }^{44}$. «El déficit moral es correlativo al intelectual y ambos obedecen a la mayor cercanía de las mujeres a la naturaleza y por tanto su limitada evolución». Por ello, «las mujeres no pueden participar ni de la educación, ni de la esfera pública en general dado el déficit intelectual y moral intrínseco a su sexo por naturaleza» ${ }^{45}$.

41. MoebiUs, P. J. La inferioridad mental de la mujer. Barcelona, Bruguera, 1982, p. 17.

42. Gómez Rodríguez, A. «Ciencia y valores en los estudios del cerebro». Arbor Ciencia pensamiento y cultura. CLXXXI, 716 (2005), p. 486.

43. GÓMEZ Rodríguez, A. Art. cit., p. 487.

44. GÓmez Rodríguez, A. Art. cit., p. 483.

45. Gómez Rodríguez, A. Art. cit., p. 487.

Feminismo/s 13, junio 2009, pp. 187-206 
$\mathrm{Al}$ anclar el argumento en la inteligencia deficiente, se pone en funcionamiento el mismo proceso de destitución de grados de humanidad que registramos con la población con discapacidad intelectual. A medida que se desciende por la escala intelectual, la falta de inteligencia va despojando al portador de la carencia de los atributos valorados del androcentrismo. Como vimos, una inteligencia deficiente trae como consecuencia fallas en la capacidad de raciocinio, de pensamiento, de juicio, de voluntad, de conciencia, de tener una conducta moral, de ser autónomos. La dominación, la sujeción o el confinamiento quedan así justificados.

Considero que la inteligencia es un operador político que atraviesa las categorías de género, raza, etnia, clase social. Los documentos históricos muestran que una y otra vez se ha argumentado e intentado comprobar una inteligencia deficiente en poblaciones de origen africano, esclavos, indígenas, clases baja, etcétera. El argumento de la deficiencia mental en diversos grupos no hegemónicos -generalmente tratados como «sexo débil», «pueblos salvajes», «pueblos primitivos», «negros», «razas inferiores», "pueblos no civilizados»- permite justificar y legitimar un destino social de sujeción.

\section{Reflexiones finales}

En este artículo se ha realizado una revisión epistemológica de la discapacidad intelectual y su criterio diagnóstico principal, el déficit en la inteligencia.

A partir del análisis del discurso de los profesionales del hospital, hemos reconstruido los elementos y la cadena significante que dan forma al diagnóstico de «retraso mental». El análisis revela al diagnóstico como una técnica política legitimada por el saber y la práctica científica, que involucra dimensiones morales y epistémicas.

A lo largo del recorrido, hemos visto que la apreciación diagnóstica expone sin eufemismos su alto contenido moral y sus supuestos epistemológicos. Sólidas certezas rodean al cuadro: lo concreto, lo lento, lo bajo, lo retrasado, lo infantil, lo instintivo, lo precario, lo primitivo, lo básico, lo simple caracteriza una y otra vez, sea cual sea el profesional o el documento relevado, al cuadro diagnóstico y a los sujetos diagnosticados.

Hemos tratado de demostrar que discapacidad intelectual e institucionalización son indiscernibles dentro de la valoración diagnóstica. Descomponiendo los elementos de la valoración, hemos situado en la falta de inteligencia el inicio del encadenamiento significante que liga al «retraso mental» con la institucionalización. Este encadenamiento reitera siempre la misma sucesión que va de la falta de inteligencia a la heteronomía: déficit intelectual, déficit en pensamiento abstracto, lógico y racional, déficit en el lenguaje, bajo control 
racional, déficits en el control de los instintos, predominio de los impulsos y automatismos en el comportamiento de los individuos; afectividad primitiva, disminución o ausencia de juicio, déficit de voluntad, disminución de la capacidad moral, mayor dependencia y mayor heteronomía.

Hemos puesto de manifiesto las imprecisiones, equívocos y vaguedades que rodean a la definición de la inteligencia, tanto entre los profesionales del hospital como en la bibliografía especializada. Sin embargo, estas múltiples definiciones revelan un núcleo de significación común: en la inteligencia se pone en juego la definición misma de lo humano tal como ha sido considerada por la tradición filosófica y metafísica occidental. Y como el feminismo ha puesto de manifiesto, la definición de lo humano en nuestra tradición occidental es indisociable de los valores del androcentrismo. La inteligencia es precisamente un operador que condensa estos valores epistémicos: la razón, la moral, la conciencia, la lógica, el lenguaje, la abstracción, la voluntad, la posibilidad de ser autónomo, maduro e independiente. La falta de inteligencia equipara al portador de esta carencia a una condición infantil, primitiva o animal.

El análisis de las dimensiones epistemológicas de la inteligencia revela que más que una condición objetiva, visible, medible o cuantificable la inteligencia es una tecnología política; y más que una cualidad -que puede estar o no, que puede ser mayor o menor- la inteligencia es una categoría política que atraviesa las relaciones de género, raza, etnias, y clase social.

La revisión epistemológica de la inteligencia en el caso de la discapacidad intelectual muestra que la inteligencia opera diferenciando seres humanos y produciendo desigualdad social. Hemos mostrado que los efectos de la tecnología de la inteligencia son la justificación y legitimación de la desigualdad y dominación. Es radicando el argumento en una inteligencia deficiente como se legitima la necesidad de supervisión, de encierro, de exclusión o incluso el exterminio de grupos no-hegemónicos de la sociedad capitalista.

La inteligencia ha servido para construir un discurso sobre la desigualdad de género y validarlo. Por ello, más que demostrar que las mujeres son tan inteligentes como los varones, o los «negros» que los «blancos», es necesario desenmascarar los elementos que componen la inteligencia, sus procedimientos, técnicas y saberes.

Precisamente, la importancia que la inteligencia adquiere en el campo de la discapacidad intelectual convierte a éste en un terreno propicio para la indagación de las prácticas y discursos sobre la inteligencia. Considero que la discapacidad intelectual se revela como paradigma del funcionamiento de la 
tecnología de la inteligencia y de los modos en que ésta produce y reproduce la desigualdad y dominación.

He intentado contribuir a la politización de la inteligencia. He intentado, al mismo tiempo, contribuir a desarmar las asociaciones semánticas y categoriales, cuestionar las certezas evidentes, y fragmentar los sólidos encadenamientos significantes presentes en el campo de la discapacidad intelectual. He intentado contribuir con ello a la posibilidad de abrir un hueco, de generar un espacio donde radicar nuevas prácticas y nuevos saberes.

A la luz de lo expuesto, la crítica epistemológica se muestra como una tarea urgente en el campo de la inteligencia y su déficit; y muy especialmente en el campo de la discapacidad intelectual: uno de los mayores problemas en el «retraso mental» es epistemológico; y por ende, profundamente político.

\section{Referencias bibliográficas}

AgAmben, G. Homo Sacer I. El poder soberano y la nuda vida. Madrid, Editorial Nacional, 2002.

- Homo Sacer III. Lo que queda de Auschwitz. Madrid, Editorial Nacional, 2002.

BOYLE, J. «Intelligence, reasoning and language proficiency». The Modern Language Journal, 71 (1987).

KelleR, E. F. Reflexiones sobre género y ciencia. Valencia, Alfons el Magnanim, 1991.

Gómez Rodríguez, A. «Ciencia y valores en los estudios del cerebro». Arbor Ciencia pensamiento y cultura. CLXXXI, 716 (2005), pp. 479-492.

GoodeY, C. F. «Intellectual ability and speed of performance: Galen to Galton». Science History Publications, XLII, (2004).

GUZMÁN CÁCERES, M. y PÉRez MAYO, A. R. «Epistemologías feministas: hacia una reconciliación política de la ciencia a través de la filosofía y la teoría de género». En BlázQUez GRAF, N. y FloRES, J. (ed), Ciencia, tecnología y género en Iberoamérica. México, Centro de Investigaciones Interdisciplinarias en Ciencias y Humanidades, Universidad Nacional Autónoma de México, 2005, pp. 635-652.

HARDING, Sandra. Ciencia y Feminismo. Madrid, Morata, 1996.

HOLLingWORTH, L. S. «Vocabulary as a symptom of intellect». American Speech, 1, 3 (1925), pp. 154-158.

Hormigo, A. K. Retraso mental en niños y adolescentes. Buenos Aires, Noveduc, 2006.

Hunt, E. «The status of the concept of intelligence». Japanese Psychological Research, 39, 1 (1997), pp. 1-11.

Lentini, E. Discapacidad Mental. Un análisis del discurso psiquiátrico. Buenos Aires, Lugar Editorial, 2007. 
MAFFIA, D. «Conocimiento y emoción», Arbor, CLXXI, 716 (2005), Madrid, noviembre-diciembre.

- «El vínculo crítico entre género y ciencia». S/d, s/a.

MARTínez FERRETI, J. «Breve guía diagnóstica y pronóstica de los retrasos mentales». Alcmeon, Revista Argentina de Clínica Neuropsiquiátrica, 8, 2 (1999), pp. 157-173.

Moebius, P. J. La inferioridad mental de la mujer. Barcelona, Bruguera, 1982.

Perez Sedeño, E. y AlCalá Cortijo, P. (coords.). Ciencia y Género. Madrid, Universidad Complutense de Madrid, 2001.

TARRIDA, A. C. Inteligencias. Una integración multidisciplinaria. Barcelona, MASSON, 2001.

WesChler, D. La medida de la inteligencia del adulto. Buenos Aires, Ed. Preliminar, s/a. 